\title{
Effects of innovative virtual reality game and EMG biofeedback on neuromotor control in cerebral palsy
}

\author{
Ji Won Yoo ${ }^{\mathrm{a}}$, Dong Ryul Lee ${ }^{\mathrm{b}}$, Yon Ju Sim ${ }^{\mathrm{a}}$, Joshua H. You, ${ }^{\mathrm{a}, *}$ and Cheol J. Kim ${ }^{\mathrm{c}}$ \\ ${ }^{a}$ Department of Physical Therapy, Yonsei University, Wonju City, Kangwon-do, Republic of South \\ Korea \\ ${ }^{b}$ Department of Physical Therapy, Honam University, Gwangju, Republic of South Korea \\ ${ }^{c}$ NeuroMedi Inc, Daejeon, Republic of South Korea
}

\begin{abstract}
Sensorimotor control dysfunction or dyskinesia is a hallmark of neuromuscular impairment in children with cerebral palsy (CP), and is often implicated in reaching and grasping deficiencies due to a neuromuscular imbalance between the triceps and biceps. To mitigate such muscle imbalances, an innovative electromyography (EMG)-virtual reality (VR) biofeedback system were designed to provide accurate information about muscle activation and motivation. However, the clinical efficacy of this approach has not yet been determined in children with CP. The purpose of this study was to investigate the effectiveness of a combined EMG biofeedback and VR (EMG-VR biofeedback) intervention system to improve muscle imbalance between triceps and biceps during reaching movements in children with spastic CP. Raw EMG signals were recorded at a sampling rate of $1,000 \mathrm{~Hz}$, band-pass filtered between $20-450 \mathrm{~Hz}$, and notch-filtered at $60 \mathrm{~Hz}$ during elbow flexion and extension movements. EMG data were then processed using MyoResearch Master Edition 1.08 XP software. All participants underwent both interventions consisting of the EMG-VR biofeedback combination and EMG biofeedback alone. EMG analysis resulted in improved muscle activation in the underactive triceps while decreasing overactive or hypertonic biceps in the EMG-VR biofeedback compared with EMG biofeedback. The muscle imbalance ratio between the triceps and biceps was consistently improved. The present study is the first clinical trial to provide evidence for the additive benefits of VR intervention for enhancing the upper limb function of children with spastic CP.
\end{abstract}

Keywords: Virtual reality, EMG visual feedback, neuromotor control, muscle imbalance, cerebral palsy

\section{Introduction}

Sensorimotor control dysfunction or dyskinesia is a hallmark of neuromuscular impairment in children with spastic cerebral palsy (CP) and is often implicated in reaching and grasping activities during play [1]. Sensorimotor control dysfunction is characterized by a neuromuscular imbalance between extensors (e.g., triceps) and elbow flexors (e.g., biceps) during functional reaching movements [2]. Normally, motor control during reaching and grasping requires reciprocal inhibition of the antagonistic elbow flexors and facilitation of the agonistic extensors [3]. Janda first coined this

\footnotetext{
*Corresponding author: Joshua H. You, Department of Physical Therapy, Yonsei University, 1 Yonseidae Kil, Wonju City, Kangwon-do 220-710, Republic of South Korea. Tel.: 033-760-2476; Fax: 033-760-2496; E-mail: neurorehab@yonsei.ac.kr.
}

0959-2989/14/\$27.50 @ 2014 - IOS Press and the authors. 
notion of neuromuscular imbalance between the flexor and extensor systems, which often results in impairments in reaching movement [4]. Clinical electromyography (EMG) studies demonstrated altered motor recruitment patterns and hypertonic muscle activation in the elbow flexor as well as decreased activity amplitude and delayed onset in the elbow extensor during reaching in children with spastic CP [5]. Thus, restoration of normal neuromuscular imbalance or control is an important intervention strategy [4].

To improve reaching motor function in children with $\mathrm{CP}$, neurorehabilitations including neurodevelopmental technique, constraint-induced movement therapy (CIMT) [6], EMG biofeedback [7], virtual reality exercise [8], and strength training [9] have been used, but outcome studies revealed various results [10]. Among the neurorehabiliations, EMG biofeedback studies showed promising results to increase motor control since it allowed accurate visual feedback about motor recruitment of the involved arm or leg muscles (plantar flexor) in children with CP. Recently, virtual reality (VR) has been an evolving exercise program which improves upper extremity motor performance and associated neuroplasticity in children with CP [8]. Such meaningful improvements in motor function may stem from the fact that it provides a fun, motivating, functional and ecological virtual exercise games that are not afforded in conventional interventions [8]. However, compensatory movement patterns during VR exercise games were also frequently observed, including the excessive antagonist (biceps) activation in an attempt to reach for a virtual object [11].

To mitigate the motor impairment in reaching, an EMG-VR exercise system has been developed recently. This system is specifically designed to improve selective activation of the agonist (triceps) while inhibiting the compensatory overactivation of the antagonist (biceps) and to offer some fun and motivation during the intervention. However, clinical therapeutic effects of this novel EMG-VR exercise system have not yet been examined in children with CP. The specific aim of this study was hence to investigate whether EMG visual feedback training based VR could improve reciprocal inhibition (triceps/biceps) during a reaching task in children with spastic CP.

\section{Materials and methods}

\subsection{Participants}

Three children with spastic $\mathrm{CP}$ (male $=2$, mean age=9.3) were recruited from a local pediatric rehabilitation center in Gimpo city, Republic of South Korea. The children's mothers provided informed consent prior to participation. The inclusion criteria for the study were (1) a diagnosis of spastic CP, (2) manual ability classification system (MACS) level I, II, III, (3) grade 1 muscle response activity according to the Modified Ashworth Scale (MAS), and (4) ability to follow verbal commands. Exclusion criteria were (1) visual disorder, (2) cognitive disorder, (3) a history of orthopedic surgery, and (4) botulinum injection in the last 12 months. The demographic and clinical characteristics of the children who participated in this study are presented in Table 1.

Table 1

Demographic and clinical characteristics of participants

\begin{tabular}{lllllll}
\hline & Age (yrs) & Gender & Type & More affected limb & MACS level & M AS grade \\
Child 1 & 8 & Male & Quadriplegic & Right & III & G1+ \\
Child 2 & 13 & Female & Quadriplegic & Left & III & G1 \\
Child 3 & 7 & Male & Diplegic & Right & II & G0 \\
\hline
\end{tabular}

Note: MACS: Manual ability classification system, MAS: Modified Ashworth scale. 


\subsection{Experimental EMG test}

EMG amplitude data were collected before and after each EMG biofeedback and a combination of EMG biofeedback and VR intervention to examine muscle imbalance between the triceps and biceps during a reaching task. Prior to EMG electrode placement, the target skin locations were prepared by shaving and rubbing the skin using alcohol-soaked pad. The electrode, a pair of hydrogel electrodes with $\mathrm{Ag} / \mathrm{AgCl}$ surfaces and an interelectrode distance of $2 \mathrm{~cm}$, was placed on the biceps brachii and triceps brachii of the affected limb. EMG electrode placement was implemented according to Cram`s guidance [12].

Raw EMG signals were recorded at a sampling rate of 1,000 Hz, and band-pass filtered between 20 $450 \mathrm{~Hz}$, and notch-filtered at $60 \mathrm{~Hz}$ during elbow flexion and extension movement. EMG data were then processed using the MyoResearch Master Edition 1.08 XP software (Noraxon Inc., Scottsdale, AZ, USA). The root mean square (RMS) was integrated with a moving window of a $100 \mathrm{~ms}$ epoch [13, 14]. A reference voluntary contraction (RVC) was used to normalize EMG data. The EMG signals with pre- and post- intervention were represented as an RMS-processed percentage of RVC (\% RVC).

\subsection{Intervention}

The QEMG-VR biofeedback system (QEMG-4XL, Laxtha, Inc., Seoul, Republic of South Korea) was used to provide a funny, motivating, and task-oriented VR exercise via the game "Aqua". Aqua was selected as an age-appropriate game for real time visual feedback exercise designed to facilitate underactive triceps activation and reciprocally inhibit overactive biceps activation during a functional reaching motor task (e.g., elbow extension movement to catch a virtual fish). As illustrated in Figure 1, each child was instructed to sit comfortably on a chair with their shoulder flexed to $90^{\circ}$, both feet flat on the floor, and knee flexed to $90^{\circ}$ in front of the QEMG-VR system computer monitor. The children had 5 minutes for practice to become familiarized with both the EMG biofeedback and VR exercise interventions. The EMG biofeedback intervention was provided first, followed by a one-week washout period after which the QEMG-VR (Aqua game) exercise was introduced. Both interventions were given 5 times per each session, each of which lasted for 60 seconds.

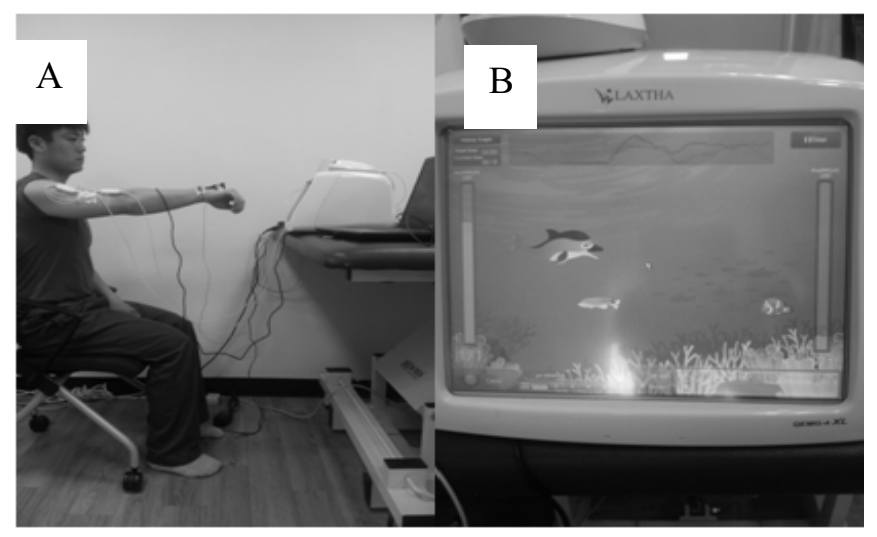

Fig. 1. Experimental setup (A) and QEMG screen (B). 
Table 2

Pre- and post-test triceps/biceps (T/B) ratio and improvement according to biofeedback method

\begin{tabular}{lllllll}
\hline & \multicolumn{3}{l}{ EMG biofeedback } & \multicolumn{3}{l}{ EMG biofeedback - VR } \\
\hline & Pretest & Posttest & Improvement (\%) & Pretest & Posttest & Improvement (\%) \\
\hline Child 1 & 0.49 & 0.05 & -89.80 & 0.29 & 0.46 & 58.62 \\
Child 2 & 0.38 & 0.27 & -28.95 & 0.12 & 2.01 & 1575.00 \\
Child 3 & 3.47 & 1.70 & -51.01 & 0.32 & 1.10 & 243.75 \\
Mean & $\mathbf{0 . 3 1}$ & $\mathbf{0 . 6 7}$ & $\mathbf{- 5 6 . 5 9}$ & $\mathbf{0 . 2 4}$ & $\mathbf{1 . 1 9}$ & $\mathbf{6 2 5 . 7 9}$ \\
\hline
\end{tabular}

Note: The ratio between Triceps and Biceps: comparing mean EMG amplitudes between triceps and biceps activity; Improvement $(\%)=[($ Posttest - Pretest $) /$ Pretest $] * 100$.

\section{Results}

\subsection{Clinical and demographic data}

The demographic and clinical characteristics of participants are presented in Table 1.

\subsection{EMG neuromuscular control data}

Table 2 shows the mean EMG amplitudes of the triceps and biceps during an elbow extension motor task. Triceps amplitude was greater during the combination of EMG biofeedback and VR (EMG-VR biofeedback) than EMG biofeedback alone. Additionally, the triceps/biceps (T/B) ratio improved more during the EMG-VR biofeedback than with EMG biofeedback, indicating that VR may have augmented the effects of EMG biofeedback.

\section{Discussion}

This is the first clinical investigation to determine the comparative effects of a combination of EMG-VR biofeedback and EMG biofeedback alone in children with spastic hemiparesis. Our results identified an additive effect of VR integrated with EMG biofeedback with respect to improving underactive triceps activation while reducing overactive biceps activation. Most importantly, our findings corroborate Janda's neuromuscular balance theory regarding sensorimotor intervention [4]. Sensorimotor control dysfunction, which is manifested by an altered neuromuscular balance between the triceps and biceps activation patterns during functional reaching movement improved more during EMG-VR than EMG alone [2]. In fact, triceps activation increased by $74.51 \%$ whereas bicep muscle was inhibited by $25.78 \%$ during the EMG-VR combination. Conversely, triceps activation decreased by $12.51 \%$ whereas biceps was activated by $190.04 \%$ during the EMG alone. This finding is consistent with the results of Lee et al., who reported increased triceps size and activation of the affected upper limb after strength training [15]. These changes were consistently reflected in gross motor performance in reaching and grasping skills [15].

The QEMG-VR biofeedback system provides not only augmented visual feedback about muscle activation, but also bring some fun and motivation for children to better engage in the virtual exercise games. Accurate augmented feedback and motivation are essential elements for motor relearning and skill acquisition in children with CP [7, 8, 16, 17]. Clinical evidence suggests that VR exercise helps children learn functional motor skills implicitly, because children are engaged in playing in any type 
of virtual environments so that they are able to develop their gross and fine motor skills and coordination naturally $[8,15-17]$.Taken together, these results support the efficacy of our novel EMGVR biofeedback intervention for enhancing the upper limb function of children with spastic CP. Improved muscle balance between the elbow flexor and extensor following the combined EMG and VR increases reaching motor performance in children with CP. The QEMG-VR system is useful for alternative biomedical and neurorehabilitation instrument in children with CP. Nevertheless, future studies should investigate the long-term effects of the QEMG-VR biofeedback system in children with $\mathrm{CP}$ as well as in other neurological populations such as hemiparetic stroke and brain injuries with a larger sample size.

\section{Acknowledgement}

Yoo and Lee have equally contributed to this project. NeuroMedi Corporation for providing the QEMG-VR system is acknowledged.

\section{References}

[1] A. Johnson, Prevalence and characteristics of children with cerebral palsy in Europe, Developmental Medicine \& Child Neurology 44 (2002), 633-640.

[2] M.G. Feltham, A. Ledebt, F.J. Deconinck and G.J. Savelsbergh, Assessment of neuromuscular activation of the upper limbs in children with spastic spastic quadriplegic cerebral palsy during a dynamical task, Journal of Electromyography \& Kinesiology 20 (2010), 448-456.

[3] R. Katz, A. Penicaud and A Rossi, Reciprocal inhibition between elbow flexors and extensors in the human, The Journal of Physiology 437 (1991), 269-286.

[4] V. Janda, Evaluation of muscular imbalance, in: Rehabilitation of the Spine, C. Frank, ed., Lippincott Williams \& Wilkins, Baltimore, 1996, pp. 97-112.

[5] M. Rassafiani and R. Sahaf, Hypertonicity in children with cerebral palsy: A new perspective, Iranian Rehabilitation Journal 11 (2011), 66-84.

[6] A.M. Gordon, Y.C. Hung, M. Brandao, C.L. Ferre, H.C. Kuo and K. Friel, Bimanual training and constraint-induced movement therapy in children with hemiplegic cerebral palsy a randomized trial, Neurorehabilitation \& Neural Repair 25 (2011), 692-702.

[7] E. Dursun, N. Dursun and D. Alican, Effects of biofeedback treatment on gait in children with cerebral palsy, Disability \& Rehabilitation 26 (2004), 116-120.

[8] S.H. You, S.H. Jang, Y.H. Kim, Y.H. Kwon, I. Barrow and M. Hallett, Cortical reorganization induced by virtual reality therapy in a child with spastic quadriplegic cerebral palsy, Developmental Medicine \& Child Neurology 47 (2005), 628-635.

[9] D.S. Marigold and J.E. Misiaszek, Whole-body responses: Neural control and implications for rehabilitation and fall prevention, The Neuroscientist 15 (2009), 36-46.

[10] A. Scianni, J.M. Butler, L. Ada and L.F. Teixeira-Salmela, Muscle strengthening is not effective in children and adolescents with cerebral palsy: A systematic review, Australian Journal of Physiotherapy 55 (2009), 81-87.

[11] H. Bateni, A. Zecevic, W.E. McIlroy and B.E. Maki, Resolving conflicts in task demands during balance recovery: Does holding an object inhibit compensatory grasping? Experimental Brain Research 157 (2004), 49-58.

[12] J. Cram, Electrode placements, in: Introduction to Surface Electromyography, G. Kasman, ed., Jones \& Bartlett Publishers, 1998, USA, pp. 371-375.

[13] P.C. Doerschuk, D.E. Gustafon and A.S. Willsky, Upper extremity limb function discrimination using EMG signal analysis, Biomedical Engineering 1(1983), 18-29.

[14] E.P. Doheny, M.M. Lowery, D.P. FitzPatrick, and M.J. O’Malley, Effect of elbow joint angle on force-EMG relationships in human elbow flexor and extensor muscles, Journal of Electromyography and Kinesiology 18.5 (2008), 760-770. 
[15] D.R. Lee, N.G. Lee and M.Y. Jung, Strength training-induced changes in muscle size and motor improvement in bilateral schizencephaly: An experimenter-blind case report with 3-month follow-up, Korean Academy of University Trained Physical Therapy 17 (2010), 77-87.

[16] N. Mumford and P.H. Wilson, Virtual reality in acquired brain injury upper limb rehabilitation: Evidence-based evaluation of clinical research, Brain Injury 23 (2009), 179-191.

[17] Y.P. Chen, L.J. Kang, T.Y. Chuang, J.L. Doong, S.J. Lee, M.W. Tsai and W.H. Sung, Use of virtual reality to improve upper-extremity control in children with cerebral palsy: A single-subject design, Physical Therapy 87 (2007), 14411457. 